UCRL-JC-131663 Abs

\title{
ORIENTATION IMAGING MICROSCOPY INVESTIGATION OF THE COMPRESSION DEFORMATION OF A [110] TA SINGLE CRYSTAL
}

Adam J. Schwartz, Wayne E. King, Geoffrey H. Campbell, James Stölken and John Y. Shu, Chemistry and Materials Science Directorate, University of California, Lawrence Livermore National Laboratory, Livermore, CA 94550; David H. Lassila, B-Division, University of California, Lawrence Livermore National Laboratory, Livermore, CA 94550; David D. Sam, Mechanical Engineering University of California, Lawrence Livermore National Laboratory, Livermore, CA 94550; Brent L. Adams and Shilei Sun, Department of Materials Science and Engineering, Carneigie Mellon University, Pittsburgh, PA

High-purity tantalum single crystal cylinders oriented with [110] parallel to the cylinder axis were deformed 10,20, and 30 percent in compression. The samples were subsequently sectioned for characterization using Orientation Imaging Microscopy (OIM) along two orthogonal sectioning planes: one in the plane containing [001] and [110] (longitudinal) and the other in the plane containing $[1 \overline{1} 0]$ and $[110]$ (transverse).

To examine local lattice rotations, the Euler angles relative to a reference angle at the section center were decomposed to their in-plane and out-of-plane components. The in-plane and out-of-plane misorientation maps for all compression tests reveal inhomogeneous deformation everywhere and particularly large lattice rotations in the corners of the longitudinal section. Of particular interest are the observed alternating orientation changes. This suggests the existence of networks of dislocations with net alternating sign that are required to accommodate the observed rotations.

Rotation maps from the transverse section are distinctly different in appearance from those in the longitudinal plane. However, the rotation maps confirm that the rotations observed above were about the [1 10$]$ axis. Alternating orientation changes are also observed on this section. Results will be directly compared with crystal rotations predicted using finite element methods and reviewed in light of the LLNL Multiscale Materials Modeling Program.

This work is performed under the auspices of U.S. Department of Energy and Lawrence Livermore National Laboratory under contract No. W-7405-Eng-48. 